\title{
Genome-wide in silico screen for CCCH-type zinc finger proteins of Trypanosoma brucei, Trypanosoma cruzi and Leishmania major
}

\author{
Susanne Kramer*, Nicola C Kimblin and Mark Carrington
}

\begin{abstract}
Background: $\mathrm{CCCH}$ type zinc finger proteins are RNA binding proteins with regulatory functions at all stages of mRNA metabolism. The best-characterized member, tritetraproline (TTP), binds to AU rich elements in 3' UTRs of unstable mRNAs, mediating their degradation. In kinetoplastids, CCCH type zinc finger proteins have been identified as being involved in the regulation of the life cycle and possibly the cell cycle. To date, no systematic listing of CCCH proteins in kinetoplastids is available.

Results: We have identified the complete set of $\mathrm{CCCH}$ type zinc finger proteins in the available genomes of the kinetoplastid protozoa Trypanosoma brucei, Trypanosoma cruzi and Leishmania major. One fifths (20\%) of all CCCH motifs fall into non-conventional classes and many had not been previously identified. One third of all CCCH proteins have more than one CCCH motif, suggesting multivalent RNA binding. One third have additional recognizable domains. The vast majority are unique to Kinetoplastida or to a subgroup within. Two exceptions are of interest: the putative orthologue of the mRNA nuclear export factor Mex67 and a 3'-5' exoribonuclease restricted to Leishmania species. CCCH motifs are absent from these proteins in other organisms and might be unique, novel features of the Kinetoplastida homologues. Of the others, several have a predicted, and in one case experimentally confirmed, connection to the ubiquitination pathways, for instance a HECT-type E3 ubiquitin ligase. The total number of kinetoplastid CCCH proteins is similar to the number in higher eukaryotes but lower than in yeast. A comparison of the genomic loci between the Trypanosomatidae homologues provides insight into both the evolution of the $\mathrm{CCCH}$ proteins as well as the CCCH motifs.

Conclusion: This study provides the first systematic listing of the Kinetoplastida $\mathrm{CCCH}$ proteins. The number of $\mathrm{CCCH}$ proteins with more then one $\mathrm{CCCH}$ motif is larger than previously estimated, due to the identification of nonconventional CCCH motifs. Experimental approaches are now necessary to examine the functions of the many unique $\mathrm{CCCH}$ proteins as well as the function of the putative Mex67 and the Leishmania 3'-5' exoribonuclease.
\end{abstract}

\section{Background}

Pathogenic kinetoplastid protozoa, such as the widely studied 'Tritryps' Trypanosoma cruzi (Tc), Leishmania major (Lm) and Trypanosoma brucei (Tb), have complex biphasic life cycles and consequently require changes in gene expression in response to extrinsic and intrinsic signals. For instance, at least $5 \%$ of all $\mathrm{Tb}$ genes are developmentally regulated at the mRNA level between any two of the experimentally tractable life cycle stages [1-4]. Kine-

*Correspondence: sk503@cam.ac.uk

1 Department of Biochemistry, University of Cambridge, Tennis Court Road, Cambridge CB2 10W, UK

Full list of author information is available at the end of the article toplastids regulate protein coding gene expression almost exclusively at the post-transcriptional level with the aid of RNA binding proteins (reviewed in [5]). One group of RNA binding proteins is defined by the presence of a $\mathrm{CCCH}$ type zinc finger motif that directly binds to RNA. Different $\mathrm{CCCH}$ proteins regulate all stages of mRNA life, amongst the best-studied are the proteins of the TIS11 family, with the best characterized being the mammalian protein tritetraproline (TTP). TIS11 proteins bind to AU-rich elements in the 3' UTRs of their target mRNAs, in most cases mediating their degradation (reviewed in [6]). The likely mechanism is the recruitment of mRNA degradation factors to the target mRNAs, 
many of which have been found to interact with TIS11 proteins [7-9]. Other $\mathrm{CCCH}$ proteins control the translation of their target mRNAs, for instance the C. elegans protein POS-1 [10,11]. The Drosophila $\mathrm{CCCH}$ protein $\mathrm{ZC} 3 \mathrm{H} 3$ regulates mRNA adenylation and nuclear export and also binds to known nuclear export factors [12]. Five Arabidopsis $\mathrm{CCCH}$ proteins have been shown to possess intrinsic endonuclease activity, including the orthologue to the polyadenylation specificity factor CPSF30 $[13,14]$. $\mathrm{CCCH}$ proteins have between 1 and $6 \mathrm{CCCH}$ motifs. These were originally defined as $\mathrm{C}-\mathrm{X}_{6-14}-\mathrm{C}-\mathrm{X}_{4-5}-\mathrm{C}-\mathrm{X}_{3}-\mathrm{H}$ [15] but recently redefined as $\mathrm{C}-\mathrm{X}_{4-15}-\mathrm{C}-\mathrm{X}_{4-6}-\mathrm{C}-\mathrm{X}_{3}-\mathrm{H}$, following the genome wide analysis of the rice and Arabidopsis $\mathrm{CCCH}$ proteins [16].

As part of a project that aimed to understand the regulation of nuclear export in trypanosomes, a putative orthologue to the yeast nuclear export factor Mex67 was identified in T. brucei. The finding of a $\mathrm{CCCH}$ motif in the putative Mex67 prompted us to set out to identify and compare the entire set of $\mathrm{CCCH}$ proteins in the genomes of the Kinetoplastida. A previous screen for the two most common $\mathrm{CCCH}$ motifs $\left(\mathrm{C}-\mathrm{X}_{7-} \mathrm{C}-\mathrm{X}_{5}-\mathrm{C}-\mathrm{X}_{3}-\mathrm{H}\right.$ and $\mathrm{C}-\mathrm{X}_{8} \mathrm{C}-$ $\left.\mathrm{X}_{5}-\mathrm{C}-\mathrm{X}_{3}-\mathrm{H}\right)$ in the Tritryp genomes identified of 50,68 , $41 \mathrm{CCCH}$ proteins in $\mathrm{Tb}$, Tc and Lm, respectively $[17,18]$. In addition, some proteins containing one of the common $\mathrm{CCCH}$ motifs also contained a $\mathrm{C}-\mathrm{X}_{10}-\mathrm{C}-\mathrm{X}_{5}-\mathrm{C}-\mathrm{X}_{3}-\mathrm{H}$ motif $[17,18]$. For ease of reading here, $\mathrm{CCCH}$ motifs in the two most common classes $\mathrm{C}-\mathrm{X}_{7-} \mathrm{C}-\mathrm{X}_{5}-\mathrm{C}-\mathrm{X}_{3}-\mathrm{H}$ and $\mathrm{C}-\mathrm{X}_{8-} \mathrm{C}-\mathrm{X}_{5}-$ $\mathrm{C}-\mathrm{X}_{3}-\mathrm{H}$ will be called 'conventional'; others, such as $\mathrm{C}$ $\mathrm{X}_{10-} \mathrm{C}-\mathrm{X}_{5}-\mathrm{C}-\mathrm{X}_{3}-\mathrm{H}$, 'non-conventional'. This term is used to highlight a difference and does not mean that they are less likely to be $\mathrm{CCCH}$ motifs [15].

Only three of the $\mathrm{CCCH}$ zinc finger proteins were identified as having readily apparent orthologues in other organisms: the splicing factor U2AF35 [19] and two components of the mRNA cleavage and polyadenylation apparatus, CPSF30 and FIP1 [20,21]. Of the previously identified $\mathrm{CCCH}$ proteins unique to kinetoplastids, two families have been experimentally characterized: (i) the ZFP CCCH proteins involved in the regulation of differentiation [22-25] and (ii) the cycle sequence binding proteins (CSBPs) that bind a conserved sequence in S-phase regulated mRNAs [26-28]. The vast majority of the trypanosome $\mathrm{CCCH}$ proteins defined by the conventional $\mathrm{CCCH}$ motifs appeared to have only one $\mathrm{CCCH}$ finger; while nearly two thirds of the Arabidopsis and rice proteins have at least two [16]. The binding of the $\mathrm{CCCH}$ protein TTP to AU rich elements is dependent on two intact $\mathrm{CCCH}$ motifs, one is not sufficient [29], and it has been speculated that in trypanosomes such multivalent RNA binding may be achieved by oligomerization, such as occurs between the $\mathrm{CCCH}$ proteins of the ZFP family $[24,30]$.
Here, an extended analysis of the $\mathrm{CCCH}$ type zinc finger proteins in the genomes of the Tritryps is presented. The inclusion of non-conventional $\mathrm{CCCH}$ motifs into the search increased the fraction of $\mathrm{CCCH}$ proteins with more than one $\mathrm{CCCH}$ motif to one third and resulted in the identification of many novel $\mathrm{CCCH}$ proteins. One example is the putative orthologue to the nuclear export factor Mex67 that has no $\mathrm{CCCH}$ motifs in mammals or fungi.

\section{Results and Discussion Identification of $\mathrm{CCCH}$ proteins in kinetoplastids}

The Tritryp genome database [31] was searched for all proteins containing the motif $\mathrm{C}-\mathrm{X}_{4-15}-\mathrm{C}-\mathrm{X}_{4-6}-\mathrm{C}-\mathrm{X}_{3}-\mathrm{H}$. This motif covers both the conventional $\left(C-X_{7}-C-X_{5}-C\right.$ $\mathrm{X}_{3}-\mathrm{H}$ and $\left.\mathrm{C}-\mathrm{X}_{8}-\mathrm{C}-\mathrm{X}_{5}-\mathrm{C}-\mathrm{X}_{3}-\mathrm{H}\right)$ and the more recently defined non-conventional $\mathrm{CCCH}$ motifs. Some motifs were recognized more than once due to multiple cysteine residues; the sequence closest to a conventional motif was used for further analysis. Protein sequences derived from genes annotated as pseudogenes in the databases were removed after the initial screen (see materials and methods for details). The resulting set contained 131, 233 and 120 proteins from $\mathrm{Tb}$, Tc and Lm, respectively, containing between one and five putative $\mathrm{CCCH}$ motifs. The larger number for Tc results from pairs of allelic variants as the genome strain (CL Brener) is a hybrid [32]. The $\mathrm{CCCH}$ motifs belonged to all possible classes, but there was a clear enrichment in the two conventional $\mathrm{CCCH}$ motifs. Of all candidate $\mathrm{CCCH}$ motifs, there were $52 / 158$ (Tb), 106/292 (Tc) and 47/148 (Lm) conventional CCCH motifs that were also recognized by one or more of SMART (Sm00356), Pfam (PF00642) or InterPro (IPR000571). These $205 \mathrm{CCCH}$ motifs (the training set) were used to produce sequence logos [Additional file 1]. There was very little difference between the sequence logos derived from each of the Tritryps [Additional file 1] and little difference between the sequence logos of the two conventional classes of $\mathrm{CCCH}$ motifs (Figure 1A and 1B). As expected for $\mathrm{CCCH}$ motifs that are recognized by Pfam, the logo was similar to the Pfam sequence logo for $\mathrm{CCCH}$ motifs or the logos for rice and Arabidopsis $\mathrm{CCCH}$ proteins [16]. A combined sequence logo was created (Figure $1 \mathrm{C}$ ) and used to evaluate all non-conventional $\mathrm{CCCH}$ motifs and conventional $\mathrm{CCCH}$ motifs not recognized by Pfam, Smart or InterPro. First, a $\mathrm{CCCH}$ motif candidate was considered a likely $\mathrm{CCCH}$ motif, if it had at least $2 / 4$ of the conserved residues coloured red in the sequence logo in Figure 1: F/Y/H/W at position $\mathrm{C} 1+3$, $\mathrm{G}$ at position $\mathrm{C} 2-2, \mathrm{G}$ at position $\mathrm{C} 3-3$ or $\mathrm{F} / \mathrm{Y} / \mathrm{Q}$ at position $\mathrm{C} 3+2$. This criterion was stringent as it excluded 5 of the 205 sequences in the training set as well as the cycling sequence binding protein A (CSBPA) of $\mathrm{Lm}$; the $\mathrm{Tb}$ and 


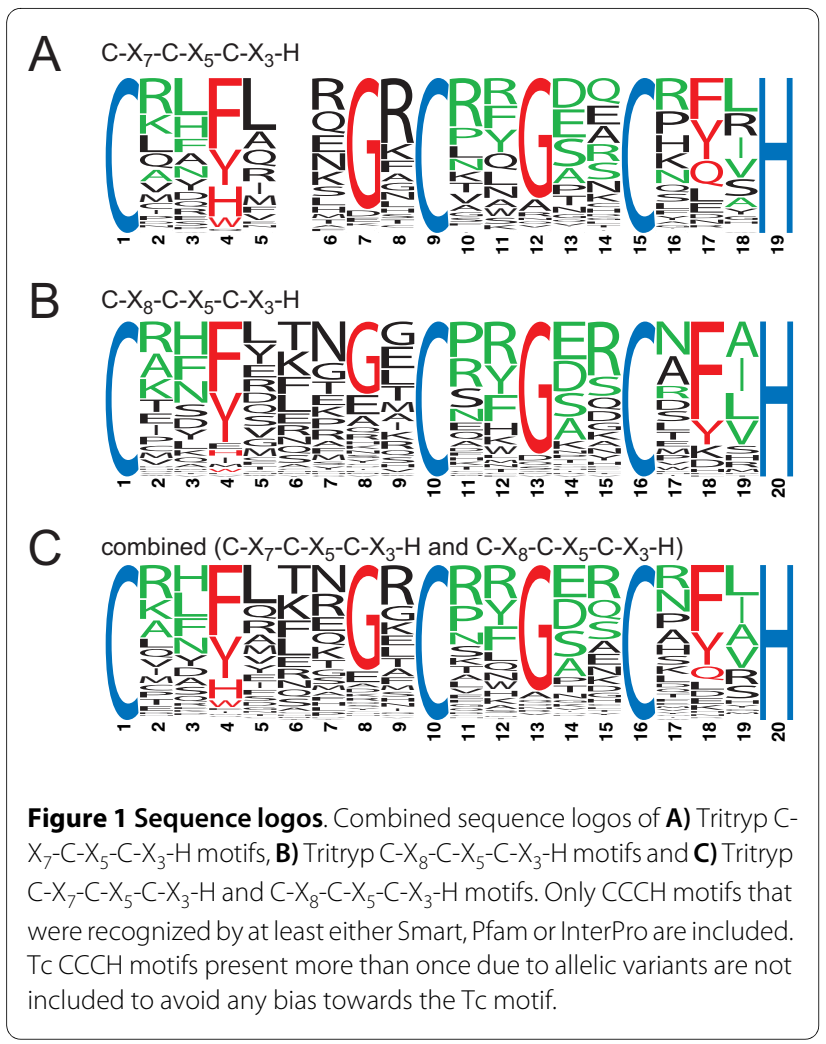

Tc orthologues of CSBPA were recognized. Therefore, in a second step, $\mathrm{CCCH}$ finger candidates not recognized by the first criterion were also considered likely $\mathrm{CCCH}$ motifs, if they had three or more identities to the amino acids coloured either red or green in the sequence logo in Figure 1. This way, all $205 \mathrm{CCCH}$ fingers of the training set and $L m C S B P A$ were included. The list now contained $66(\mathrm{~Tb}), 133(\mathrm{Tc})$, and $78(\mathrm{Lm})$ candidate $\mathrm{CCCH}$ zinc finger proteins. In a third step, proteins that were unlikely to be $\mathrm{CCCH}$ zinc finger proteins were removed manually: (i) cell surface proteins or proteins with a known function unrelated to nucleic acids and (ii) $\mathrm{CCCH}$ motif candidates with homologues in the other Tritryps that did not possess the $\mathrm{CCCH}$ motif. In most cases, the manually excluded proteins had non-conventional $\mathrm{CCCH}$ motifs that had little similarity to the sequence logo, supporting the finding that they were not real $\mathrm{CCCH}$ zinc fingers. The exceptions were five proteins that were classified as 'possible $\mathrm{CCCH}$ zinc finger proteins'. These were excluded from the final list but are discussed below. After removing allelic variants from the Tc set, the list of $\mathrm{CCCH}$ proteins contained $51(\mathrm{~Tb}), 53(\mathrm{Tc})$ and $54(\mathrm{Lm})$ sequences [Additional file 2]. These numbers were reduced to $48(\mathrm{~Tb}), 51(\mathrm{Tc})$ and $54(\mathrm{Lm})$ after $\mathrm{CCCH}$ proteins encoded by genes that were present in tandem arrays of the same genomic locus and had more than $40 \%$ amino acid sequence identity to each other (e.g. gene products of recently duplicated genes) were counted as one in order to limit redundancy. The complete list of genes is shown schematically in Figure 2. Together, the Tritryps have 62 non-redundant $\mathrm{CCCH}$ proteins. Of these, 39 are present in all three species; the remaining 23 are unique to one or two of the Tritryps (Figure 3A, Figure 2). Roughly one third, 16/48 (Tb), 20/51 (Tc), and 16/ $54(\mathrm{Lm})$, have more than one $\mathrm{CCCH}$ motif (Figure 3B, Figure 2). Many of these have one non-conventional $\mathrm{CCCH}$ motif, the reason they had not been classified previously as containing multiple $\mathrm{CCCH}$ fingers. $20 \%$ of all $\mathrm{CCCH}$ motifs were non-conventional with $\mathrm{C}-\mathrm{X}_{7} \mathrm{C}-\mathrm{X}_{4}-\mathrm{C}$ $\mathrm{X}_{3}-\mathrm{H}, \mathrm{C}-\mathrm{X}_{8}-\mathrm{C}-\mathrm{X}_{4}-\mathrm{C}-\mathrm{X}_{3}-\mathrm{H}$ and the previously identified C$\mathrm{X}_{10}-\mathrm{C}-\mathrm{X}_{5}-\mathrm{C}-\mathrm{X}_{3}-\mathrm{H}$ being the most abundant non-conventional $\mathrm{CCCH}$ motifs (Figure 3C) [Additional File 2]. About one third of all $\mathrm{CCCH}$ proteins, 14/48 (Tb), 15/51 (Tc) and 18/54 (Lm), have other recognizable domains (Figure 2). In addition to the domains present in the experimentally characterized $\mathrm{CCCH}$ proteins described above, recognizable domains included other zinc finger domains (ZnF_C2H2, Zf-RanBP, ZnF_HIT), a cytidine deaminase domain, a helicase domain, a DNAJ domain, an exonuclease domain, a HECT domain and a U-box. The vast majority of $\mathrm{CCCH}$ proteins are unique to Kinetoplastida, or even to a subgroup of the Kinetoplastida. Most have no similarities to proteins of other eukaryotes; some have similarities that are restricted to conserved domains only. Two exceptions were a putative Mex67 orthologue and a Leishmania specific 3 ' exoribonuclease, discussed below. Using the identity between $\mathrm{Tb}$ and $\mathrm{Lm}$ for illustration, the most conserved protein was the splicing factor U2af35 (64\%), followed by ZC3H9 (52\%), ZC3H17 (49\%), CPSF30 (48\%), ZC3H23 (48\%) and ZC3H41 (48\%) (Figure 2).

\section{A CCCH motif in a putative Mex67/TAP/NXF1}

This investigation of $\mathrm{CCCH}$ proteins was initiated by an in silico search for a trypanosome homologue of the budding yeast mRNA export factor Mex67 (NXF1 and TAP in mammalian cells, reviewed in [33]). Using standard BLAST parameters, the protein encoded by Tb11.22.0004 gave the highest p-value (2.3e-07) and screening the $S$. cerevisiae proteome with Tb11.22.0004 gave a single hit, Mex67, p-value (1.5e-07) [Additional file 3:Supplemental Figure S2A]. An InterPro search for domains and motifs in Tb11.22.0004 detected the presence of PTHR10662 [34], characteristic of NXF1-related proteins, and a $\mathrm{CCCH}$ zinc finger near the $\mathrm{N}$-terminus. $S$. cerevisiae Mex67 and mammalian NXF1 do not contain a zinc finger [Additional file 3:Supplemental Figure S2A].

Members of the NXF1 family are generally not very similar to each other, for example, the region of highest identity between the Drosophila melanogaster NXF1 and S. cerevisiae Mex67 (residues 107-598, determined by NCBI blast2seq) is $23 \%$ identical. Using the same pro- 


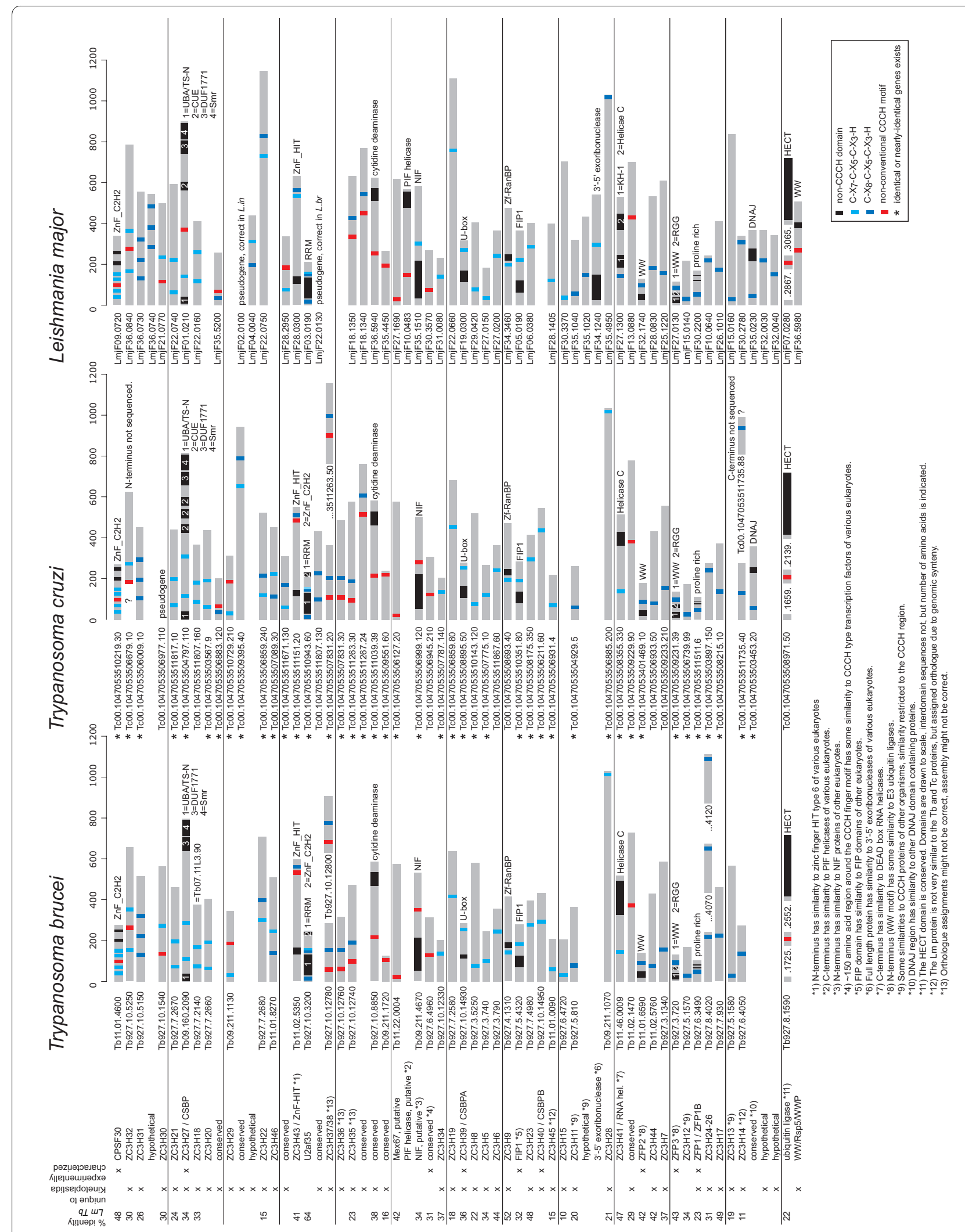

Figure $\mathbf{2} \mathrm{CCCH}$ proteins of the Tritryps. All CCCH proteins of the final list are shown to scale. Conventional (blue) and non-conventional (red) $\mathrm{CCCH}$ motifs as well as any recognizable domains are indicated. The identities between the Tb and $\mathrm{Lm}$ homologues (first column) were calculated using the needle algorithm of Emboss pairwise alignments. 

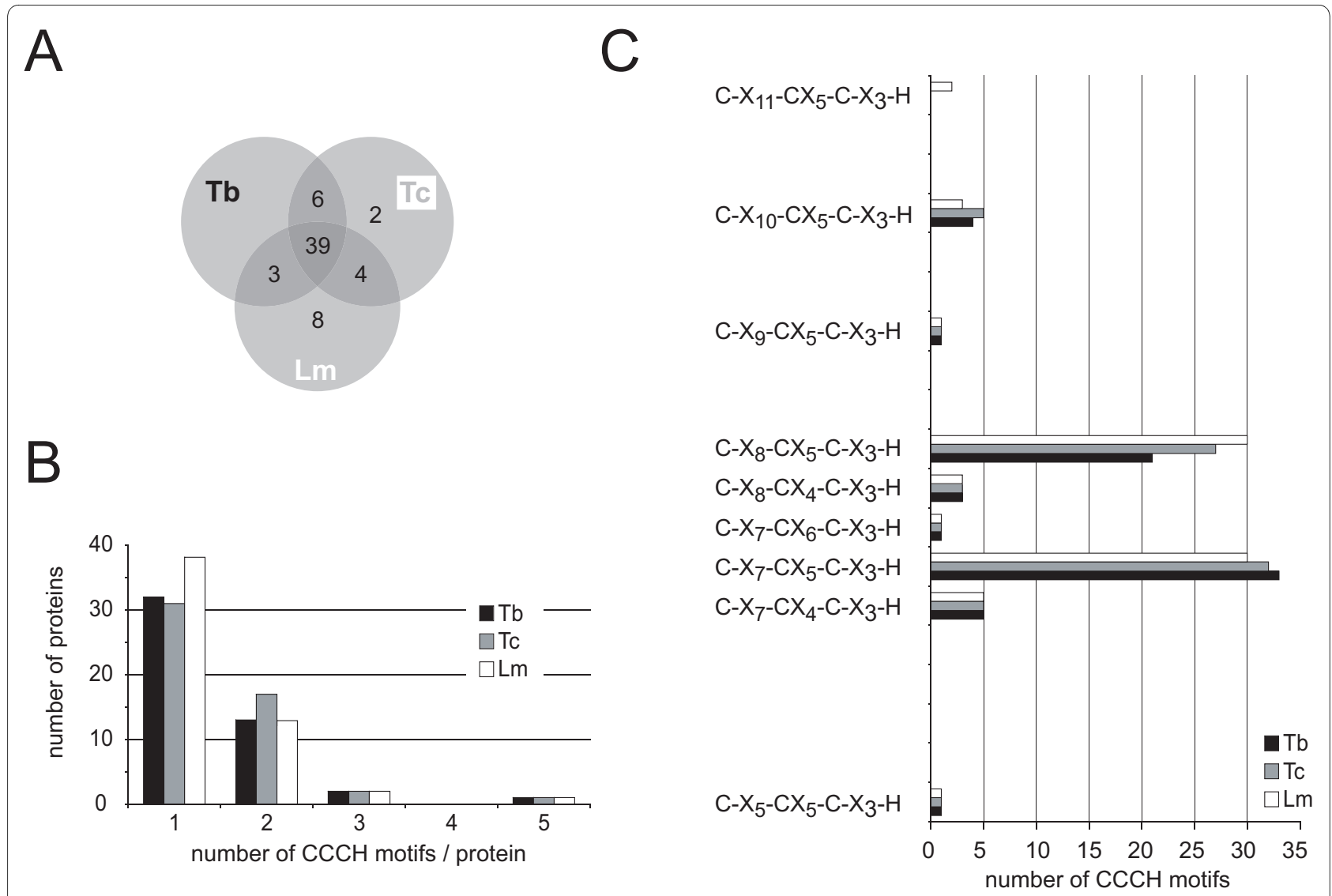

Figure 3 Statistics on kinetoplastid CCCH proteins. A) Numbers of CCCH proteins present in all Tritryps or the respective subset. (B) Numbers of $\mathrm{CCCH}$ proteins with $1,2,3$, or $5 \mathrm{CCCH}$ motifs. C) Numbers of $\mathrm{CCCH}$ motifs per $\mathrm{CCCH}$-motif-class.

gramme, the region of highest identity between $S$. cerevisiae Mex67 and Tb11.22.0004 was identified as $\sim 160$ amino acids at the $\mathrm{N}$-termini (Mex67 residues 96-248 and Tb11.22.0004 residues 49 to 206) and had 31\% identity. The same identity was found between Drosophila NXF1 and S. cerevisiae Mex67 for the same region [Additional file 3:Supplemental Figure S2B]. The closest homologues of $\mathrm{Tb} 11.22 .004$ in the other Tritryps, Tc00.1047053506127.20/Tc00.1047053508271.4 and LmjF27.1690 also contain a zinc finger near the N-terminus and have closest homology to the yeast mRNA nuclear export factor Mex67 and its mammalian orthologue TAP/NXF1.

The subcellular localisation of the putative Tb MEX67 was determined by fluorescent protein tagging of the endogenous locus of $\mathrm{Tb} 11.02 .0004$. The protein localized to the nucleoplasm as well as to spots at the nuclear periphery that are similar in number and size to spots seen with an eYFP fusion of the nucleoporin NUP54/57 (Figure 4A). The localization of Mex67 to nuclear pores has been described for both yeast Mex67 [35] and the mammalian homologue TAP [36]. This observation sup- ports, but does not prove, the identification of Tb11.02.0004 as the Mex67 orthologue.

The finding that the putative kinetoplastid MEX67 contains an RNA-binding zinc finger is novel and Mex67 homologues from other non-Opisthokonta species were investigated. Neither the Mex67 homologues from Dictyostelium discoideum nor from Entamoeba histolytica (both Amoebozoa) contained a $\mathrm{CCCH}$ domain. No close Mex67 homologue was readily recognisable in the available genome sequences of organisms in either the Archaeplastida (plants) or the Chromalveolata (Figure 4B). Furthermore, other than in kinetoplastids, Mex67 homologues were not readily recognisable in other Excavata species: Naegleria gruberi, Giardia lamblia and Trichomonas vaginalis. The role of the $\mathrm{CCCH}$ motif in the putative trypanosome MEX67 remains unknown, but it might indicate differences in the regulation of mRNA nuclear export between trypanosomes and other organisms.

\section{A CCCH protein with a 3' exoribonuclease domain}

The Leishmania-specific 3' exoribonuclease, LmjF34.1240, is similar to 3 ' exoribonucleases of various 


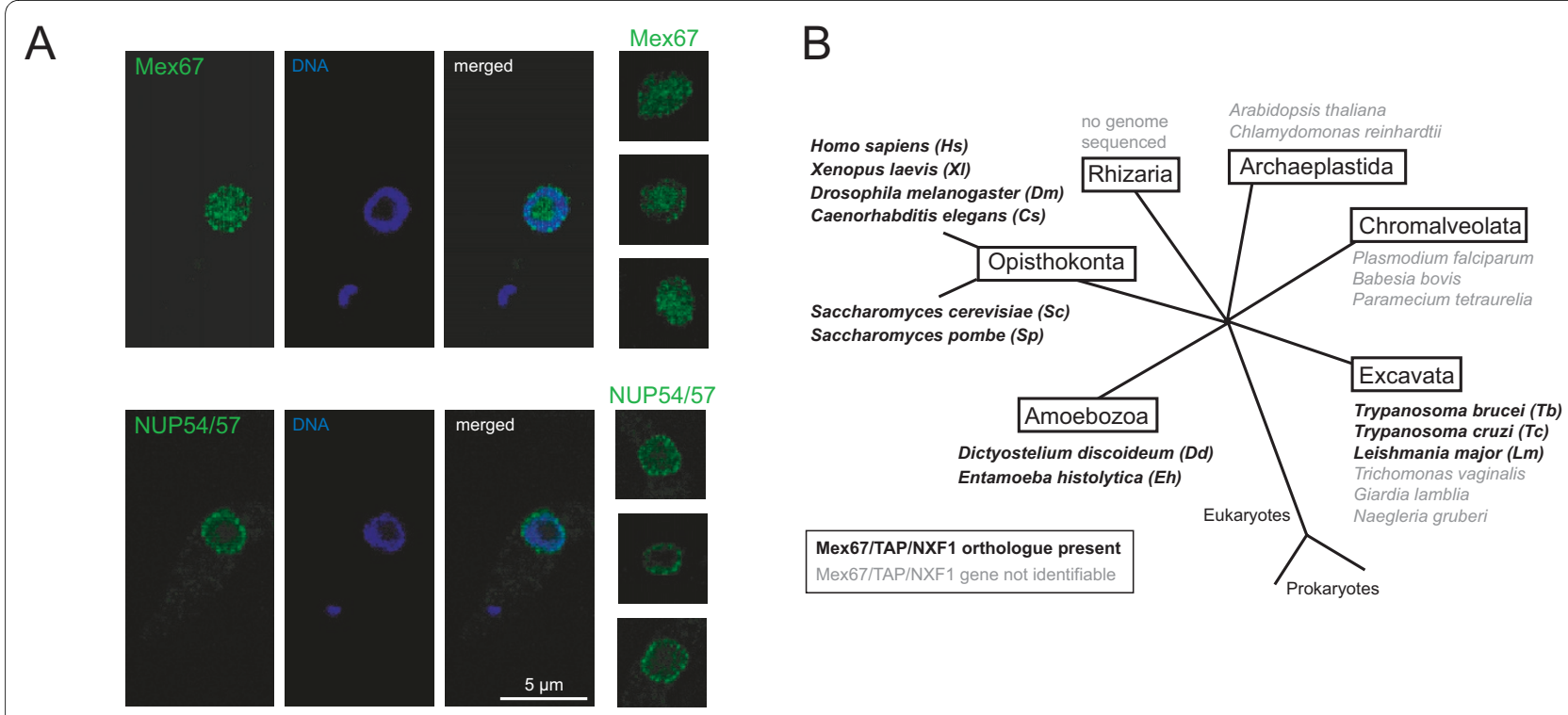

Figure 4 Mex67/TAP/NXF1. A) A C-terminally tagged eYFP fusion protein of the T. brucei putative Mex67 orthologue (Tb11.22.0004) was expressed from its endogenous locus in procyclic trypanosomes. Single plane confocal microscopy images are shown. For comparison, single plane confocal microscopy images of cells expressing an eYFP fusion protein of the nucleoporin NUP54/57 are shown. B) Available genomes of organisms that are representative for five of the six major groups of the eukaryotic kingdom were searched for proteins homologous to the S. cerevisiae Mex 67 by Blast. Organisms that have an identifiable Mex67 gene are shown in black/bold, organisms without a readily identifiable Mex67 homologue are shown in grey.

eukaryotes and the region of homology is not restricted to the exoribonuclease domain but extends over the entire protein [Additional file 4]. The $\mathrm{CCCH}$ motif, however, is unique to the Leishmania protein. Several Arabidopsis $\mathrm{CCCH}$ proteins possess intrinsic nuclease activity, including CPSF30 and Smic1 [13,14]. However, neither of these proteins has a clearly defined nuclease domain and the nuclease activity was dependent on one (CPSF30) or two (Smic1) of the CCCH motifs. To our knowledge, the Leishmania protein is the first protein that has both a $3^{\prime}$ exoribonuclease domain and a $\mathrm{CCCH}$ motif and it would be very interesting to examine whether the $\mathrm{CCCH}$ motif is involved in regulating exoribonuclease activity and/or substrate specificity. The exoribonuclease is present in all Leishmania species, but absent from all trypanosome species. It is tempting so speculate that the enzyme might be involved in mRNA regulation via the cis-acting element SIDER (Short Interspersed DEgenerated Retrotransposon). SIDER elements are mainly found in $3^{\prime}$ UTRs of Leishmania genes, where they promote mRNA degradation (SIDER2, [37]) or regulate translation $[38,39]$. In contrast, SIDER elements are 70 times less abundant in $\mathrm{Tb}$ and usually found in the subtelomeric regions [37] and do not appear to function as regulatory cis-acting elements of mRNAs.

\section{$\mathrm{CCCH}$ finger proteins and ubiquitination}

Ubiquitination requires the ubiquitin-activating enzyme (E1), the ubiquitin conjugating enzyme (E2) and the ubiq- uitin protein ligase (E3). Substrate specificity is usually determined by the E3 ligase. Three different types of E3 ligases can be distinguished, named after their catalytic domains: the RING type, the (RING-related) U-box type and the HECT type. Substrates can either be monoubiquitinated, multi-ubiquitinated (monoubiquitinated on multiple lysine residues) or polyubiquitinated (carrying a chain of ubiquitins). Depending on the type and place of ubiquitination, ubiquitinated proteins can be either targeted for degradation by the $26 \mathrm{~S}$ proteosome or the ubiquitination acts as a signal, for example to change the intracellular localization of the protein.

Among the kinetoplastid $\mathrm{CCCH}$ proteins are several with a predicted, and in one case experimentally confirmed, connection to ubiquitination. Both ZFP2 and ZFP3 have a motif upstream of the WW domain that has closest homology to a motif upstream of the WW domain of HECT type E3 ligases of the Nedd4 family [22,24] (Figure 2). CSBP (ZC3H27) has two different types of ubiquitin interacting domains: UBA (ubiquitin associated domain) and CUE [28] (Figure 2). UBA domains have highest affinity for polyubiquitin; CUE domains, in contrast, have been shown to bind to monoubiquitin and promote autoubiquitination (reviewed in [40]). In fact, a fraction of the Leishmania CSBP protein has been shown to be monoubiquitinated and a small fraction is either polyubiquitinated or multi-monoubiquitinated [28], consistent with the CUE domain mediating autoubiquitination. Two putative E3 ubiquitin ligases are among the 
Kinetoplastida $\mathrm{CCCH}$ finger proteins: one U-box type and one HECT-type E3 ubiquitin ligase (Figure 2).

Proteins with a combination of domains associated with ubiquitination and RNA binding are common in many species [41]; trypanosomes are no exception. Ubiquitination may play important roles in the regulation of stability or localization of RNA binding proteins. C. elegans oogenesis and embryogenesis provide two good examples: five germline specific $\mathrm{CCCH}$ proteins (PIE-1, POS-1, MEX-1, MEX-5, MEX06) are degraded in somatic cells via binding of the $\mathrm{CCCH}$-finger binding protein ZIF-1 to the CCCH motif and recruitment of an E3 ubiquitin ligase [42]. Oma-1, a $\mathrm{CCCH}$ protein involved in asymmetric distribution of determinants in the egg is degraded during the first zygotic cell cycle via a ZIF-1independent E3 ubiquitin ligase complex [43].

Four of the trypanosome $\mathrm{CCCH}$ proteins that have a predicted or known connection to ubiquitination are involved in the regulation of the life cycle or cell cycle. In one case, CSBP, ubiquitination of the $\mathrm{CCCH}$ protein has been shown. It is possible that ubiquitination is a mechanism to quickly change either stability or intracellular localization of $\mathrm{CCCH}$ proteins in response to life and/or cell cycle triggers.

\section{Origin of the kinetoplastid $\mathrm{CCCH}$ proteins}

Most $\mathrm{CCCH}$ proteins are present in all three Tritryps (Figure 3A); the average amino acid sequence identity between the closest homologues in $\mathrm{Tb}$ and $\mathrm{Lm}$ is $32 \%$ (Figure 2). Nevertheless, there are variations in the $\mathrm{CCCH}$ protein content between the Tritryps (Figure 2) and to examine the origin of these differences, the synteny between the genomic loci coding for the $\mathrm{Tb}$ and the $\mathrm{Lm} \mathrm{CCCH}$ proteins was examined.

The $\mathrm{CCCH}$ proteins of $\mathrm{Tb}$ and $\mathrm{Lm}$ are distributed over 47 genomic loci (Figure 5). 27 of these loci contain single $\mathrm{CCCH}$ proteins; of these, 19 have full synteny, seven have synteny with minor changes close to the locus and one has synteny to one side only (Figure 5A). Another 11 loci contain tandemly repeated genes in $\mathrm{Tb}$ and/or $\mathrm{Lm}$, with at least one pair between the species (Figure 5B-E). These 11 fall into four different groups: two loci have full synteny with significantly higher similarity between the homologous pairs than between the duplicated genes within the locus, indicating that gene duplication took place before the separation of $\mathrm{Tb}$ and Lm (Figure 5B). Three loci have a duplicated gene present in only one of the species, but with significantly higher similarity between the homologues from the two species than between the duplicated genes, probably best explained by loss of one of the duplicated genes from one species after the separation of $\mathrm{Tb}$ and $\mathrm{Lm}$ (Figure $5 \mathrm{C}$ ). One locus has three identical copies of a $\mathrm{Tb}$ gene, while the syntenic $\mathrm{Lm}$ locus has only one gene with $31 \%$ identity to the $\mathrm{Tb}$ genes, indicating that the gene duplication probably occurred after divergence (Figure 5D). The remaining five loci also contain differences between the two species in the number of duplicated genes, but it remains unclear, whether these originated from a gene duplication event in one of the species or from the loss of a gene in the other (Figure 5E).

Nine loci have a $\mathrm{CCCH}$ protein gene in only one of the two species (Figure 5F and 5G). For eight loci, regional synteny is still intact, usually with one or two other genes out of synteny in addition to the gene coding for the $\mathrm{CCCH}$ proteins (Figure 5F). In one case, synteny was lost (Figure 5G). Are the differences between these loci due to the loss or the gain of the gene in one of the species? Four of the Lm genes are also present in Tc, indicating that the loss of the $\mathrm{Tb}$ gene is the more likely scenario, since Leishmanias separated from Trypanosomes before the separation of $\mathrm{Tb}$ and Tc. Four of the $\mathrm{Lm}$ genes are absent from both $\mathrm{Tc}$ and $\mathrm{Tb}$, suggesting that they either arose after the separation of Leishmania from the trypanosomes or were lost after the separation of Leishmania in the common ancestor of $\mathrm{Tc}$ and $\mathrm{Tb}$. One gene is present in $\mathrm{Tb}$ and $\mathrm{Tc}$, but not in $\mathrm{Lm}$, indicating that it was either lost in Leishmania, or gained in the common ancestor of $\mathrm{Tc}$ and $\mathrm{Tb}$.

Taken together, the analysis confirms that the majority of the kinetoplastid $\mathrm{CCCH}$ proteins genes evolved prior to the separation of the species and the presence of many loci with more than one $\mathrm{CCCH}$ protein gene suggests that gene duplication was important in the evolution of $\mathrm{CCCH}$ proteins. Differences in the content of the genes coding for $\mathrm{CCCH}$ proteins between $\mathrm{Tb}$ and $\mathrm{Lm}$ can be accounted for by (i) the loss or gain of a single gene or (ii) gene duplication or loss of a previously duplicated gene; in both cases this occurred with only small changes in synteny.

\section{Possible $\mathrm{CCCH}$ proteins}

Five proteins were classified as "possible $\mathrm{CCCH}$ proteins" and excluded from the final list, because the $\mathrm{CCCH}$ motif was absent from one or both of the other Tritryps. Alignments are shown in Figure 6 and give some insight into how $\mathrm{CCCH}$ motifs might have evolved. One Leishmania protein (LmjF34.0870) has a large insertion in the middle of its $\mathrm{CCCH}$ motif, while the $\mathrm{CCCH}$ motifs of $\mathrm{Tb}$ and $\mathrm{Tc}$ are intact (Figure 6A). It is unlikely that the Leishmania protein contains a zinc finger. $\mathrm{A} \mathrm{Tb}$ protein (Tb927.3.1610) has a $\mathrm{CCCH}$ motif in the middle of a kinase domain; the motif is absent from the other Tritryps despite of the high degree of conversion in this region (Figure 6B). Similarly, the previously classified $\mathrm{Tb}$ zinc finger protein $\mathrm{ZC} 3 \mathrm{H} 16$ (Tb927.7.250) has a conventional $\mathrm{CCCH}$ motif that is absent from the closest homologues in Tc and Lm (Figure 6C). In this case, the selective loss or 


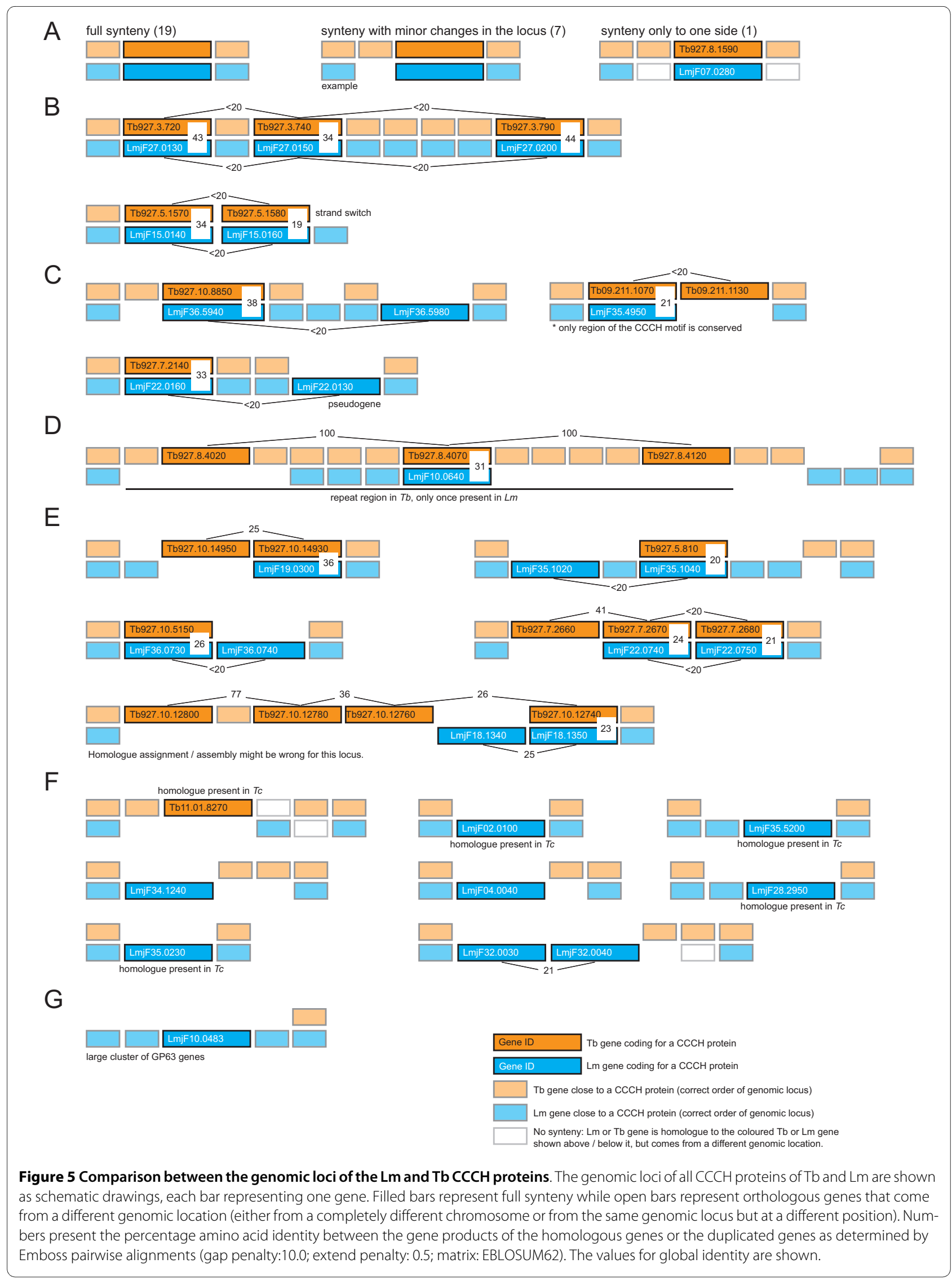




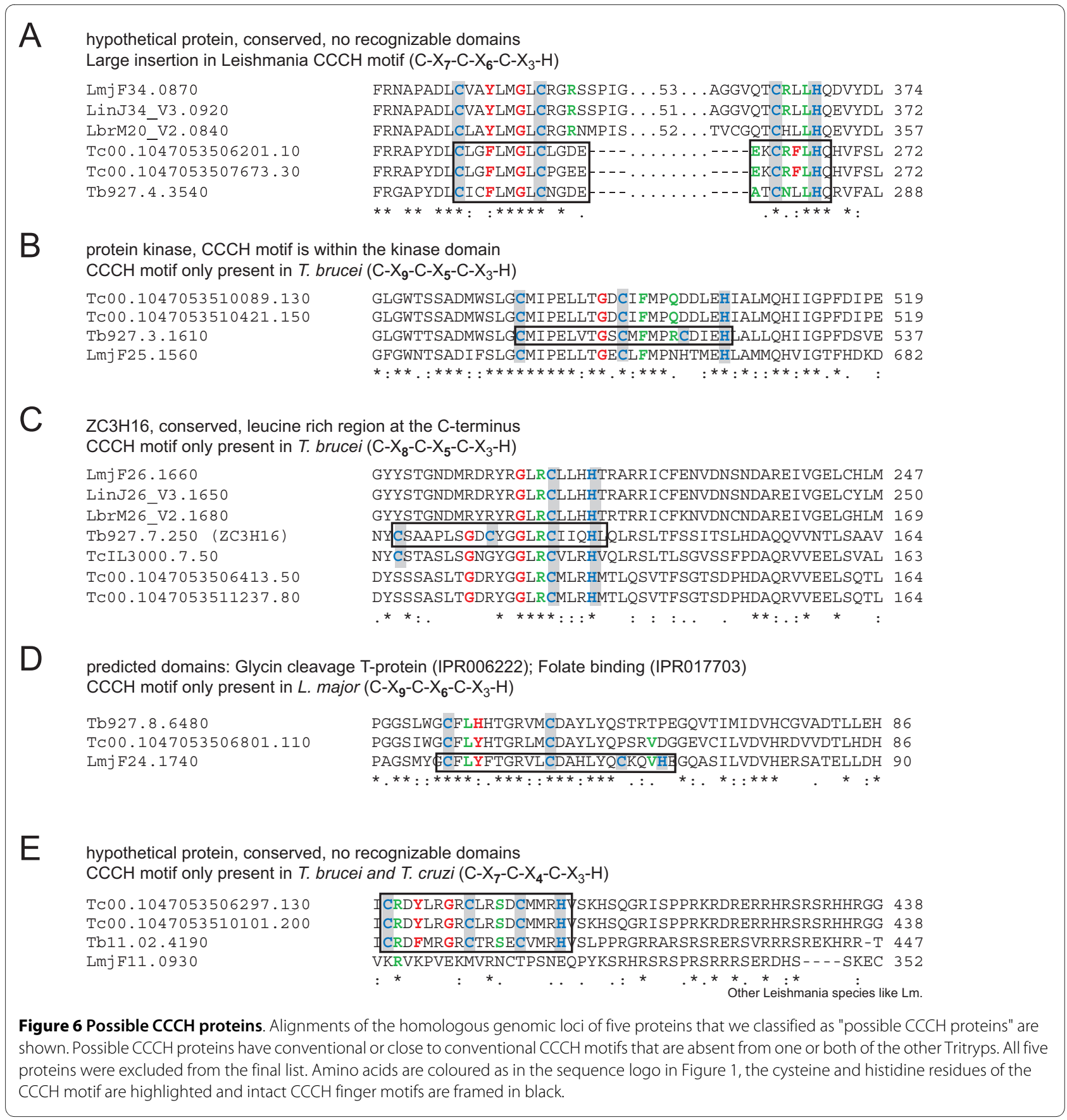

gain of a $\mathrm{CCCH}$ motif in evolution is clearly visible: $T$. congolense, a close relative to $\mathrm{Tb}$, has two cysteine residues of the $\mathrm{CCCH}$ motif, while Lm and Tc have only one. A similar case for a Leishmania protein ( $\mathrm{LmjF} 24.1740)$ is shown in Figure 6D. In another case, a C-X $7-C-X_{4}-C-X_{3}-$ $\mathrm{H}$ motif is present in $\mathrm{Tb}$ and $\mathrm{Tc}$ (Tb11.02.4190; Tc00.1047053506297.130; Tc00.1047053510101.200), but absent from Leishmania (LmjF11.0930). However, the Leishmania protein has very little homology to its $\mathrm{Tb}$ and Tc homologues in the region of the $\mathrm{CCCH}$ motif (Figure $6 \mathrm{E})$. In this case it is likely that the $\mathrm{CCCH}$ motif was lost or gained by larger genomic rearrangements and it might be fully functional in $\mathrm{Tb}$ and Tc. Without experimental data it is impossible to predict whether either of the possible proteins $\mathrm{CCCH}$ proteins have functional $\mathrm{CCCH}$ type zinc fingers.

\section{Comparison with other eukaryotes}

The number of non-redundant $\mathrm{CCCH}$ proteins in the Tritryps (48 in Tb, 51 in Tc, 54 in $\mathrm{Lm}$ ) is similar to the number in higher eukaryotes: Arabidopsis, rice, mouse and human have $68,67,58$ and 55 predicted $\mathrm{CCCH}$ pro- 
teins, respectively $[16,44]$. The fraction of $\mathrm{CCCH}$ proteins with more than one $\mathrm{CCCH}$ motif $(34 \%)$ is about half of the fraction found among the rice $(64.2 \%)$ or Arabidopsis (63.2\%) $\mathrm{CCCH}$ proteins.

It has been previously suggested that kinetoplastids have significantly more $\mathrm{CCCH}$ proteins than other lower eukaryotes, based on a comparison with yeast. In the absence of published data, we attempted an estimation by screening protozoa and yeast genomes for the two conventional $\mathrm{CCCH}$ motifs and, as a background control, for two non-existing ones $\left(\mathrm{C}-\mathrm{X}_{7}-\mathrm{C}-\mathrm{X}_{7}-\mathrm{C}-\mathrm{X}_{3}-\mathrm{H}\right.$ and $\mathrm{C}-\mathrm{X}_{8}-\mathrm{C}-$ $\left.\mathrm{X}_{7}-\mathrm{C}-\mathrm{X}_{3}-\mathrm{H}\right)$, without any filtering or evaluation steps. The results are summarized in Table 1: kinetoplastids have slightly more $\mathrm{CCCH}$ motifs than any other lower eukaryotes investigated, but they are closely followed by T. gondii and D. discoideum. Other protozoa and yeast do have fewer $\mathrm{CCCH}$ motifs. These data are an estimation, but they do suggest that the number of $\mathrm{CCCH}$ proteins in trypanosomes is only slightly higher than in some protozoa, while other protozoa and the two yeasts have fewer $\mathrm{CCCH}$ proteins.

\section{Conclusions}

This study has identified the entire set of $\mathrm{CCCH}$ proteins in the available genomes of the Tritryps; there are 48 in $\mathrm{Tb}, 51$ in Tc and 54 in Lm, excluding redundancy. The main findings are: (i) The fraction of $\mathrm{CCCH}$ proteins with more than one $\mathrm{CCCH}$ motif is larger than previously assumed; many of these proteins have one non-conventional $\mathrm{CCCH}$ motif. (ii) The putative Mex67 orthologue as well as a Leishmania-specific 3'exoribonuclease both have a $\mathrm{CCCH}$ motif that is not found in their counterparts in other eukaryotes. Many of the $\mathrm{CCCH}$ proteins have a predicted, or in one case experimentally confirmed, connection to ubiquitination pathways. (iii) Kinetoplastids do have only slightly more $\mathrm{CCCH}$ proteins than some other protozoa, although the number of $\mathrm{CCCH}$ proteins is higher than in yeast. (iv) The vast majority of the $\mathrm{CCCH}$ proteins are unique to kinetoplastids or to a subgroup within. The majority evolved before the separation of the Tritryps; gene duplication played an important role. Differences in the $\mathrm{CCCH}$ protein content between the Tritryps is mainly due to either the loss or gain of a single gene or gene duplication or loss of a previously duplicated gene; in all cases with little disruption of synteny.

The identification of $\mathrm{CCCH}$ proteins in this study relies entirely on in silico data. Some of the identified proteins might not be true $\mathrm{CCCH}$ zinc finger proteins, whilst others might have been missed. For instance, some putative $\mathrm{CCCH}$ proteins were excluded from the final list because of the absence of the $\mathrm{CCCH}$ motif in one or both of their closest homologue in one or both of the other Tritryps; they might, however, be true $\mathrm{CCCH}$ proteins. Although the majority of $\mathrm{CCCH}$ motifs bind RNA, a few examples of DNA binding $\mathrm{CCCH}$ motifs have been reported (for example [45-47]); thus, it is possible that some of the identified trypanosome $\mathrm{CCCH}$ proteins are not RNAbinding proteins.

Experimental approaches are now needed to verify the in silico data and to examine the function of the many uncharacterized proteins. Of particular interest is the function of a $\mathrm{CCCH}$ motif in the putative nuclear export

Table 1: Estimation of the numbers of conventional $\mathrm{CCCH}$ motifs in protozoa and yeast.

\begin{tabular}{|c|c|c|c|c|c|c|c|c|}
\hline \multirow[t]{2}{*}{ Organism } & No of & iffs (ur & $\overline{Y / Z}($ & -(-X3-H) & & Genome & & \multirow[t]{2}{*}{$\begin{array}{c}\text { Total number o } \\
\text { CCCH motifs } \\
\text { (background } \\
\text { corrected) }\end{array}$} \\
\hline & $7 / 5$ & $8 / 5$ & $7 / 7$ & $8 / 7$ & Size (Mbp) & No of genes & Ref. & \\
\hline T. brucei & 37 & 28 & 4 & 2 & 26.1 & 9068 & [59] & 59 \\
\hline L. major & 31 & 31 & 5 & 6 & 32.8 & 8272 & [17] & 51 \\
\hline T. cruzi & 62 & 59 & 3 & 8 & 60.4 & 22570 & {$[60]$} & 110 \\
\hline T. annulata & 7 & 6 & 0 & 1 & 8.4 & 3792 & {$[61]$} & 12 \\
\hline D. discoideum & 18 & 18 & 4 & 2 & 33.9 & 12500 & {$[62]$} & 30 \\
\hline P. falciparum & 24 & 9 & 2 & 5 & 22.9 & 5268 & {$[63]$} & 26 \\
\hline T. gondii GT1 & 36 & 19 & 5 & 1 & 63.0 & 8155 & [64]; ToxoDB & 49 \\
\hline S. pombe & 12 & 9 & 2 & 2 & 12.5 & 5027 & {$[65]$} & 17 \\
\hline S. cerevisiae & 7 & 6 & 1 & 0 & 12.1 & 5885 & {$[66]$} & 12 \\
\hline
\end{tabular}

The genomes of the organisms listed were searched for the two conventional CCCH motifs and in addition for two fantasy $\mathrm{CCCH}$ motifs (C- $\mathrm{X}_{7}$ - $\mathrm{C}$ $\mathrm{X}_{7}-\mathrm{C}-\mathrm{X}_{3}-\mathrm{H}$ and $\left.\mathrm{C}-\mathrm{X}_{8}-\mathrm{C}-\mathrm{X}_{7}-\mathrm{C}-\mathrm{X}_{3}-\mathrm{H}\right)$. No filtering steps were performed. The total number of conventional $\mathrm{CCCH}$ motifs was estimated by adding the numbers of the two conventional $\mathrm{CCCH}$ motifs and subtracting the numbers of the two fantasy $\mathrm{CCCH}$ motifs. 
factor Mex67 and in the Leishmania-specific 3'-5' exoribonuclease. Both $\mathrm{CCCH}$ motifs are unique features of the Kinetoplastida proteins and might reveal differences to other eukaryotes in mRNA metabolism.

\section{Methods \\ Software}

Sequence logos were produced using the software of [48]. All analyses of the Tritryp genomes were performed using the tools at either the Tritryps genome database [31], GeneDB http://www.genedb.org/Homepage or EBI. Sequence alignments of multiple sequences were done using ClustalW2 with default settings as provided by the server ([49]; http://www.ebi.ac.uk/Tools/clustalw2/ index.html). Pairwise alignments were performed using the EMBOSS Needle programme http://www.ebi.ac.uk/ Tools/emboss/align/index.html[50] for global alignments and the Water programme [51] or BLAST2seq for local alignments. Identification of protein domains was by Pfam [52], SMART [53,54], InterPro [55] or Prosite [56].

\section{Identification of $\mathrm{CCCH}$ proteins}

The Tritryp genome databases [31] were searched for $\mathrm{CCCH}$ motif containing proteins using motif search for $\mathrm{C}-\mathrm{X}_{4-15}-\mathrm{C}-\mathrm{X}_{4-6}-\mathrm{C}-\mathrm{X}_{3}-\mathrm{H}$. A sequence logo was created including only $\mathrm{C}-\mathrm{X}_{7 / 8}-\mathrm{C}-\mathrm{X}_{5}-\mathrm{C}-\mathrm{X}_{3}-\mathrm{H}$ motifs that were recognized by at least either SMART (Sm00356), Pfam (PF00642) or InterPro (IPR000571) (the training set) and are thus very likely to be real $\mathrm{CCCH}$ motifs. This consensus motif was then used to arbitrarily define conditions to further filter all $\mathrm{CCCH}$ motifs that did not fall into the group of the training set. The stringency of the chosen conditions was tested on the training set and gradually decreased until it included all proteins in the training set. The dataset was further filtered manually to exclude proteins unlikely to contain $\mathrm{CCCH}$ motifs using the criteria described in the results section.

\section{Identification of pseudogenes}

The dataset contained four genes that were annoted as pseudogenes in the genome databases, two in L. major (LmjF02.0100 and LmjF22.0130) and two in T. cruzi (Tc00.1047053506977.110 and Tc00.1047053511715.50). Both Leishmania pseudogenes have premature stop codons and longer counterparts in other Leishmania strains (see Figure 2). Confirmation of the sequence for the Leishmania major isoforms was obtained from Matt Rogers (Sanger institute). Tc00.1047053506977.110 also has a premature stop codon and a longer counterpart in T. congolense. Tc00.1047053511715.50 has an internal shift out of frame and then back in again, the $\mathrm{CCCH}$ motif is in the out of frame region and therefore an artefact.

\section{Intracellular localization of Mex67}

The putative T. brucei Mex67 orthologue (Tb11.22.0004) and NUP54/57 (Tb927.4.5200) were expressed in Trypanosoma brucei Lister 427 procyclic cells as C-terminally tagged eYFP fusion proteins from their endogenous loci as described in [57]. For microscopic imaging, cells were washed once in SDM79 without serum or haem, fixed at a density of $1 * 10^{7}$ cells/ml with $2.4 \%$ paraformaldehyde overnight, washed once in PBS and stained with Hoechst H33258. Confocal images were prepared using a BioRad Radiance 2100 on a Nikon Eclipse E800 upright microscope using a 100/1.4 Oil DIC objective. Transgenic trypanosomes were generated using standard procedures [58].

\section{List of Abbreviations}

Tb: Trypanosoma brucei; Lm: Leishmania major; Tc: Trypanosoma cruzi; Lb: Leishmania braziliensis; Li: Leishmania infantum; Tc:Trypanosoma congolense; CSBP: cycling sequence binding protein.

\section{Additional material}

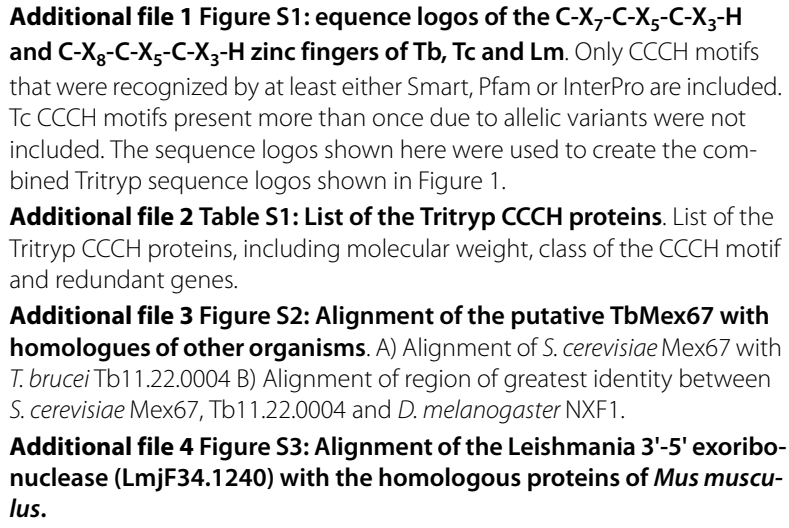

Additional file 2 Table S1: List of the Tritryp $\mathrm{CCCH}$ proteins. List of the Tritryp CCCH proteins, including molecular weight, class of the $\mathrm{CCCH}$ motif and redundant genes.

Additional file 3 Figure S2: Alignment of the putative TbMex67 with homologues of other organisms. A) Alignment of S. cerevisiae Mex67 with T. brucei Tb1 1.22.0004 B) Alignment of region of greatest identity between S. cerevisiae Mex67, Tb11.22.0004 and D. melanogaster NXF1.

Additional file 4 Figure S3: Alignment of the Leishmania 3'-5' exoribonuclease (LmjF34.1240) with the homologous proteins of Mus musculus.

\section{Authors' contributions}

All experimental and in silico work was done by SK. The manuscript was prepared by SK and MC. NCK cloned and characterized the T. brucei Mex67 protein. All authors approved to the content of the manuscript.

\section{Acknowledgements}

This work was funded by the Wellcome Trust. NCK held a Medical Research Council PhD studentship. We would like to thank Matt Rogers (Sanger institute) for help with the identification of Leishmania pseudogenes.

\section{Author Details}

Department of Biochemistry, University of Cambridge, Tennis Court Road, Cambridge CB2 1QW, UK

Received: 4 February 2010 Accepted: 5 May 2010

Published: 5 May 2010

\section{References}

1. Kabani S, Fenn K, Ross A, Ivens A, Smith TK, Ghazal P, Matthews K: Genome-wide expression profiling of in vivo-derived bloodstream parasite stages and dynamic analysis of mRNA alterations during synchronous differentiation in Trypanosoma brucei. BMC Genomics 2009, 10:427. 
2. Jensen BC, Sivam D, Kifer CT, Myler PJ, Parsons M: Widespread variation in transcript abundance within and across developmental stages of Trypanosoma brucei. BMC Genomics 2009, 10:482.

3. Queiroz R, Benz C, Fellenberg K, Hoheisel JD, Clayton C: Transcriptome analysis of differentiating trypanosomes reveals the existence of multiple post-transcriptional regulons. BMC Genomics 2009, 10:495.

4. Brems S, Guilbride DL, Gundlesdodjir-Planck D, Busold C, Luu V-D, Schanne M, Hoheisel J, Clayton C: The transcriptomes of Trypanosoma brucei Lister 427 and TREU927 bloodstream and procyclic trypomastigotes. Mol Biochem Parasitol 2005, 139(2):163-172.

5. Clayton C, Shapira M: Post-transcriptional regulation of gene expression in trypanosomes and leishmanias. Mol Biochem Parasitol 2007, 156(2):93-101.

6. Baou M, Jewell A, Murphy JJ: TIS11 family proteins and their roles in posttranscriptional gene regulation. J Biomed Biotech 2009 2009:634520

7. Lykke-Andersen J, Wagner E: Recruitment and activation of mRNA decay enzymes by two ARE-mediated decay activation domains in the proteins TTP and BRF-1. Genes Dev 2005, 19(3):351-361.

8. Fenger-Gron M, Fillman C, Norrild B, Lykke-Andersen J: Multiple processing body factors and the ARE binding protein TTP activate mRNA decapping. Mol Cell 2005, 20(6):905-915.

9. Chen CY, Gherzi R, Ong SE, Chan EL, Raijmakers R, Pruijn GJ, Stoecklin G, Moroni C, Mann M, Karin M: AU binding proteins recruit the exosome to degrade ARE-containing mRNAs. Cell 2001, 107(4):451-464.

10. Ogura K, Kishimoto N, Mitani S, Gengyo-Ando K, Kohara Y: Translational control of maternal glp-1 mRNA by POS-1 and its interacting protein SPN-4 in Caenorhabditis elegans. Development 2003, 130(11):2495-2503.

11. Tabara H, Hill RJ, Mello CC, Priess JR, Kohara Y: pos-1 encodes a cytoplasmic zinc-finger protein essential for germline specification in C. elegans. Development 1999, 126(1):1-11.

12. Hurt JA, Obar RA, Zhai B, Farny NG, Gygi SP, Silver PA: A conserved CCCHtype zinc finger protein regulates mRNA nuclear adenylation and export. J Cell Biol 2009, 185(2):265-277.

13. Addepalli B, Hunt AG: A novel endonuclease activity associated with the Arabidopsis ortholog of the $30-\mathrm{kDa}$ subunit of cleavage and polyadenylation specificity factor. Nucleic Acids Res 2007, 35(13):4453-4463.

14. Addepalli B, Hunt AG: Ribonuclease activity is a common property of Arabidopsis CCCH-containing zinc-finger proteins. FEBS Lett 2008, 582(17):2577-2582.

15. Berg JM, Shi Y: The galvanization of biology: a growing appreciation for the roles of zinc. Science 1996, 271(5252):1081-1085.

16. Wang D, Guo Y, Wu C, Yang G, Li Y, Zheng C: Genome-wide analysis of $\mathrm{CCCH}$ zinc finger family in Arabidopsis and rice. BMC Genomics 2008, 9:44.

17. Ivens AC, Peacock CS, Worthey EA, Murphy L, Aggarwal G, Berriman M, Sisk E, Rajandream MA, Adlem E, Aert R, et al:: The genome of the kinetoplastid parasite, Leishmania major. Science 2005, 309(5733):436-442.

18. Hendriks E, Matthews K: Post-Transcriptional Control of Gene Expression in African Trypanosomes. Trypanosomes After the Genome 2007:209-237.

19. Vazquez M, Atorrasagasti C, Bercovich N, Volcovich R, Levin MJ: Unique features of the Trypanosoma cruzi U2AF35 splicing factor. Mol Biochem Parasitol 2003, 128(1):77-81.

20. Hendriks EF, Abdul-Razak A, Matthews KR: tbCPSF30 depletion by RNA interference disrupts polycistronic RNA processing in Trypanosoma brucei. J Biol Chem 2003, 278(29):26870-26878.

21. Bercovich N, Levin MJ, Vazquez MP: The FIP-1 like polyadenylation factor in trypanosomes and the structural basis for its interaction with CPSF30. Biochem Biophys Res Commun 2009, 380(4):850-855.

22. Hendriks EF, Robinson DR, Hinkins M, Matthews KR: A novel CCCH protein which modulates differentiation of Trypanosoma brucei to its procyclic form. Embo J 2001, 20(23):6700-6711.

23. Hendriks EF, Matthews KR: Disruption of the developmental programme of Trypanosoma brucei by genetic ablation of TbZFP1, a differentiation-enriched CCCH protein. Mol Microbio/ 2005, 57(3):706-716.

24. Paterou A, Walrad P, Craddy P, Fenn K, Matthews K: Identification and stage-specific association with the translational apparatus of TbZFP3, a
$\mathrm{CCCH}$ protein that promotes trypanosome life-cycle development. J Biol Chem 2006, 281(51):39002-39013.

25. Walrad P, Paterou A, Acosta-Serrano A, Matthews KR: Differential trypanosome surface coat regulation by a $\mathrm{CCCH}$ protein that $\mathrm{Co}-$ associates with procyclin mRNA cis-elements. PLoS Pathog 2009, 5(2):e1000317.

26. Mahmood R, Hines JC, Ray DS: Identification of cis and trans elements involved in the cell cycle regulation of multiple genes in Crithidia fasciculata. Mol Cell Biol 1999, 19(9):6174-6182.

27. Mahmood R, Mittra B, Hines JC, Ray DS: Characterization of the Crithidia fasciculata mRNA cycling sequence binding proteins. Mol Cell Biol 2001, 21(14):4453-4459.

28. Bhandari D, Saha P: mRNA cycling sequence binding protein from Leishmania donovani (LdCSBP) is covalently modified by ubiquitination. FEMS Microbiol Lett 2007, 273(2):206-213.

29. Lai WS, Carballo E, Strum JR, Kennington EA, Phillips RS, Blackshear PJ: Evidence that tristetraprolin binds to AU-rich elements and promotes the deadenylation and destabilization of tumor necrosis factor alpha mRNA. Mol Cell Biol 1999, 19(6):4311-4323.

30. Caro F, Bercovich N, Atorrasagasti C, Levin MJ, Vázquez MP: Protein interactions within the TcZFP zinc finger family members of Trypanosoma cruzi: implications for their functions. Biochemical and Biophysical Research Communications 2005, 333(3):1017-1025.

31. Aslett M, Aurrecoechea C, Berriman M, Brestelli J, Brunk BP, Carrington M, Depledge DP, Fischer S, Gajria B, Gao X, et al.: TriTrypDB: a functional genomic resource for the Trypanosomatidae. Nucleic Acids Res 2010:D457-462.

32. Machado CA, Ayala FJ: Nucleotide sequences provide evidence of genetic exchange among distantly related lineages of Trypanosoma cruzi. Proc Natl Acad Sci USA 2001, 98(13):7396-7401.

33. Carmody SR, Wente SR: mRNA nuclear export at a glance. J Cell Sci 2009, 122(Pt 12):1933-1937

34. Thomas PD, Campbell MJ, Kejariwal A, Mi H, Karlak B, Daverman R, Diemer K, Muruganujan A, Narechania A: PANTHER: a library of protein families and subfamilies indexed by function. Genome Res 2003, 13(9):2129-2141.

35. Segref A, Sharma K, Doye V, Hellwig A, Huber J, Luhrmann R, Hurt E: Mex67p, a novel factor for nuclear mRNA export, binds to both poly(A)+ RNA and nuclear pores. Embo J 1997, 16(11):3256-3271.

36. Katahira J, Strasser K, Podtelejnikov A, Mann M, Jung JU, Hurt E: The Mex67p-mediated nuclear mRNA export pathway is conserved from yeast to human. Embo J 1999, 18(9):2593-2609.

37. Bringaud F, Müller M, Cerqueira GC, Smith M, Rochette A, El-Sayed NMA Papadopoulou B, Ghedin E: Members of a large retroposon family are determinants of post-transcriptional gene expression in Leishmania. PLOS Pathog 2007, 3(9):1291-1307.

38. Boucher N, Wu Y, Dumas C, Dube M, Sereno D, Breton M, Papadopoulou $B$ : A common mechanism of stage-regulated gene expression in Leishmania mediated by a conserved 3'-untranslated region element. J Biol Chem 2002, 277(22):19511-19520

39. McNicoll F, Müller M, Cloutier S, Boilard N, Rochette A, Dubé M, Papadopoulou B: Distinct 3'-untranslated region elements regulate stage-specific mRNA accumulation and translation in Leishmania. Biol Chem 2005, 280(42):35238-35246.

40. Hurley JH, Lee S, Prag G: Ubiquitin-binding domains. Biochem J 2006, 399(3):361-372.

41. Anantharaman V, Koonin EV, Aravind L: Comparative genomics and evolution of proteins involved in RNA metabolism. Nucleic Acids Res 2002, 30(7):1427-1464

42. DeRenzo C, Reese KJ, Seydoux G: Exclusion of germ plasm proteins from somatic lineages by cullin-dependent degradation. Nature 2003, 424(6949):685-689.

43. Shirayama M, Soto MC, Ishidate T, Kim S, Nakamura K, Bei Y, Heuvel S van den, Mello CC: The Conserved Kinases CDK-1, GSK-3, KIN-19, and MBK-2 Promote OMA-1 Destruction to Regulate the Oocyte-to-Embryo Transition in C. elegans. Curr Biol 2006, 16(1):47-55.

44. Liang J, Song W, Tromp G, Kolattukudy PE, Fu M: Genome-wide survey and expression profiling of $\mathrm{CCCH}$-zinc finger family reveals a functional module in macrophage activation. PLoS One 2008, 3(8):e2880.

45. Hwang ES, Choi A, Ho IC: Transcriptional regulation of GATA-3 by an intronic regulatory region and fetal liver zinc finger protein $1 . J$ Immunol 2002, 169(1):248-253. 
46. Wang L, Xu Y, Zhang C, Ma Q, Joo SH, Kim SK, Xu Z, Chong K: OsLIC, a Novel CCCH-Type Zinc Finger Protein with Transcription Activation, Mediates Rice Architecture via Brassinosteroids Signaling. PLoS One 2008, 3(10):e3521

47. Pomeranz MC, Hah C, Lin PC, Kang SG, Finer JJ, Blackshear PJ, Jang JC: The Arabidopsis tandem zinc finger protein AtTZF1 traffics between the nucleus and cytoplasmic foci and binds both DNA and RNA. Plant Physio/ 152(1):151-165.

48. Crooks GE, Hon G, Chandonia JM, Brenner SE: WebLogo: a sequence logo generator. Genome Res 2004, 14(6):1188-1190.

49. Larkin MA, Blackshields G, Brown NP, Chenna R, McGettigan PA, McWilliam $H$, Valentin F, Wallace IM, Wilm A, Lopez R, et al: Clustal W and Clustal X version 2.0. Bioinformatics 2007, 23(21):2947-2948.

50. Needleman SB, Wunsch CD: A general method applicable to the search for similarities in the amino acid sequence of two proteins. $\mathrm{J} \mathrm{Mol} \mathrm{Biol}$ 1970, 48(3):443-453.

51. Smith TF, Waterman MS: Identification of common molecular subsequences. J Mol Biol 1981, 147(1):195-197.

52. Finn RD, Tate J, Mistry J, Coggill PC, Sammut SJ, Hotz HR, Ceric G, Forslund K, Eddy SR, Sonnhammer EL, et al:: The Pfam protein families database. Nucleic Acids Res 2008:D281-288.

53. Schultz J, Milpetz F, Bork P, Ponting CP: SMART, a simple modular architecture research tool: identification of signaling domains. Proc Natl Acad Sci USA 1998, 95(11):5857-5864.

54. Letunic I, Doerks T, Bork P: SMART 6: recent updates and new developments. Nucleic Acids Res 2009:D229-232.

55. Hunter S, Apweiler R, Attwood TK, Bairoch A, Bateman A, Binns D, Bork P, Das U, Daugherty L, Duquenne L, et al.: InterPro: the integrative protein signature database. Nucleic Acids Res 2009:D211-215.

56. Hulo N, Bairoch A, Bulliard V, Cerutti L, Cuche BA, de Castro E, Lachaize C, Langendijk-Genevaux PS, Sigrist CJ: The 20 years of PROSITE. Nucleic Acids Res 2008:D245-249.

57. Kelly S, Reed J, Kramer S, Ellis L, Webb H, Sunter J, Salje J, Marinsek N, Gull K, Wickstead B, et al:: Functional genomics in Trypanosoma brucei: a collection of vectors for the expression of tagged proteins from endogenous and ectopic gene loci. Mol Biochem Parasitol 2007, 154(1):103-109.

58. McCulloch R, Vassella E, Burton P, Boshart M, Barry JD: Transformation of monomorphic and pleomorphic Trypanosoma brucei. Methods Mol Biol 2004, 262:53-86

59. Berriman M, Ghedin E, Hertz-Fowler C, Blandin G, Renauld H, Bartholomeu DC, Lennard NJ, Caler E, Hamlin NE, Haas B, et al:: The genome of the African trypanosome Trypanosoma brucei. Science 2005, 309(5733):416-422

60. El-Sayed NM, Myler PJ, Bartholomeu DC, Nilsson D, Aggarwal G, Tran AN, Ghedin E, Worthey EA, Delcher AL, Blandin G, et al: The genome sequence of Trypanosoma cruzi, etiologic agent of Chagas disease. Science 2005, 309(5733):409-415.

61. Pain A, Renauld H, Berriman M, Murphy L, Yeats CA, Weir W, Kerhornou A, Aslett M, Bishop R, Bouchier C, et al:: Genome of the host-cell transforming parasite Theileria annulata compared with T. parva. Science 2005, 309(5731):131-133.

62. Eichinger L, Pachebat JA, Glockner G, Rajandream MA, Sucgang R, Berriman M, Song J, Olsen R, Szafranski K, Xu Q, et al:: The genome of the social amoeba Dictyostelium discoideum. Nature 2005, 435(7038):43-57.

63. Gardner MJ, Hall N, Fung E, White O, Berriman M, Hyman RW, Carlton JM, Pain A, Nelson KE, Bowman S, et al:: Genome sequence of the human malaria parasite Plasmodium falciparum. Nature 2002, 419(6906):498-511.

64. Gajria B, Bahl A, Brestelli J, Dommer J, Fischer S, Gao X, Heiges M, lodice J, Kissinger JC, Mackey AJ, et al:: ToxoDB: an integrated Toxoplasma gondii database resource. Nucleic Acids Res 2008:D553-556.

65. Wood V, Gwilliam R, Rajandream MA, Lyne M, Lyne R, Stewart A, Sgouros J, Peat N, Hayles J, Baker S, et al:: The genome sequence of Schizosaccharomyces pombe. Nature 2002, 415(6874):871-880.

66. Goffeau A, Barrell BG, Bussey H, Davis RW, Dujon B, Feldmann H, Galibert F, Hoheisel JD, Jacq C, Johnston M, et al: Life with 6000 genes. Science 1996, 274(5287):546-547. doi: $10.1186 / 1471-2164-11-283$

Cite this article as: Kramer et al., Genome-wide in silico screen for $\mathrm{CCCH}$

type zinc finger proteins of Trypanosoma brucei, Trypanosoma cruzi and

Leishmania major BMC Genomics 2010, 11:283

\section{Submit your next manuscript to BioMed Central and take full advantage of:}

- Convenient online submission

- Thorough peer review

- No space constraints or color figure charges

- Immediate publication on acceptance

- Inclusion in PubMed, CAS, Scopus and Google Scholar

- Research which is freely available for redistribution

Submit your manuscript at www.biomedcentral.com/submit
C Biomed Central 